\title{
Arm Skeletal Muscle Mass Is Associated With the Prognosis of Patients With Cirrhosis
}

\author{
YOSHIHIRO SHIMONO ${ }^{1}$, HIRAYUKI ENOMOTO ${ }^{1}$, KYOHEI KISHINO ${ }^{1}$, \\ EI-ICHIRO MORIWAKI ${ }^{1,2}$, HIROKI NISHIKAWA ${ }^{1}$, TAKASHI NISHIMURA ${ }^{1}$, \\ YOSHINORI IWATA $^{1}$, HIROKO IIJIMA ${ }^{1}$ and SHUHEI NISHIGUCHI ${ }^{1}$ \\ ${ }^{1}$ Division of Hepatobiliary and Pancreatic Disease, Department of Internal Medicine, \\ Hyogo College of Medicine, Hyogo, Japan; \\ ${ }^{2}$ Department of Gastroenterology, Kano General Hospital, Osaka, Japan
}

\begin{abstract}
Background/Aim: This study aimed to assess the clinical significance of measuring the arm skeletal muscle mass in patients with cirrhosis. Patients and Methods: Using body composition data measured with the bioimpedance analysis (BIA) method, the skeletal muscle mass index (SMI) values of the arm (arm skeletal muscle mass/height ${ }^{2}$ ) and leg (leg skeletal mass muscle/height ${ }^{2}$ ) were calculated for 353 patients with cirrhosis, and the relationships of these indices to their prognosis were assessed. In addition, overhydration of the upper and lower limbs was compared. Results: Arm SMI was significantly positively associated with the prognosis of patients with cirrhosis ( $p=0.0002)$ but leg SMI was not $(p=0.0829)$. The rate of overhydration in the lower limbs was significantly higher than that in the upper limbs $(p<0.0001)$. Conclusion: Arm SMI measured with the BIA method was suggested to be minimally affected by water retention, and might be clinically useful for patients with cirrhosis.
\end{abstract}

Sarcopenia was initially reported as the loss of muscle mass in elderly individuals (1). However, sarcopenia occurs as a complication in several diseases ('secondary sarcopenia') and the presence of disease-related sarcopenia is associated with a poor prognosis (2). Although patients with chronic liver diseases (CLDs) often suffer from sarcopenia, these patients have specific disease-related clinical characteristics.

This article is freely accessible online.

Correspondence to: Hirayuki Enomoto, Division of Hepatobiliary and Pancreatic Disease, Department of Internal Medicine, Hyogo College of Medicine, Mukogawa-cho1-1, Nishinomiya, Hyogo 6638501, Japan. Tel: +81 798456472, Fax: +81 798456474, e-mail: enomoto@hyo-med.ac.jp

Key Words: Bioimpedance analysis, skeletal muscle mass index, upper limbs, liver cirrhosis, prognosis.
Thus, the Japanese Society of Hepatology proposed a guideline for the management of sarcopenia specifically for patients with CLD (3). In the guideline, the skeletal muscle mass index (SMI), which is calculated using the appendicular skeletal muscle mass determined by bioimpedance analysis (BIA), is used for the diagnosis of sarcopenia. Thus, as in other guidelines (4-8), there is no distinction between the upper and lower limbs.

Patients with liver cirrhosis (LC) tend to develop an overhydrated state $(9,10)$, and a report has suggested the usefulness of muscle mass measurement in the upper limbs alone, as the BIA-based muscle mass data in the lower limbs may be susceptible to edema (11). However, whether the skeletal muscle mass of the upper limbs and that of the lower limbs have a different clinical impact on the prognosis of patients with LC has not been clarified. In this study, we measured the upper limb skeletal muscle mass and the lower limb skeletal muscle mass separately, and evaluated their association with the prognosis of patients with LC.

\section{Patients and Methods}

Out of patients who underwent a body composition assessment using the BIA method from February 2006 to August 2011, those with LC were enrolled. The diagnosis of LC was determined based on clinical data, including the liver biopsy findings, laboratory data and imaging data (ultrasound, computed tomography and endoscopy findings) (12). In the present study, we evaluated the nutritional status of patients with LC via both direct anthropometric measurement and the BIA method on the same day. Patients whose laboratory data were obtained on the same day as the nutritional assessment were collected and a total of 353 patients were analyzed. The study was approved by the Ethics Committee of our Institutional Review Board (Approval No.: 1831).

Body composition analysis. The body composition of the patients was analyzed using the BIA method with an $\operatorname{InBody} 720^{\circledR}$ instrument (Inbody Japan, Tokyo, Japan). The BIA device can provide the skeletal muscle mass values in the right arm, left arm, right leg and 
in vivo $34: 1165-1171(2020)$

Table I. The basic clinical characteristics of the study patients $(N=353)$.

\begin{tabular}{|c|c|c|}
\hline Characteristic & Subgroup & Value \\
\hline Age, years & Median (range) & $66(24-85)$ \\
\hline \multicolumn{3}{|l|}{ Gender, n } \\
\hline Male & 211 & \\
\hline Female & 142 & \\
\hline \multicolumn{3}{|l|}{ Child-Pugh grade, $n$} \\
\hline A & 191 & \\
\hline B & 135 & \\
\hline $\mathrm{C}$ & 27 & \\
\hline \multicolumn{3}{|l|}{ Etiology, n } \\
\hline HBV & 25 & \\
\hline $\mathrm{HCV}$ & 228 & \\
\hline $\mathrm{HBV}+\mathrm{HCV}$ & 4 & \\
\hline ALD & 35 & \\
\hline NASH & 7 & \\
\hline $\mathrm{AIH}$ & 13 & \\
\hline $\mathrm{PBC}$ & 8 & \\
\hline Other & 33 & \\
\hline Body mass index, $\mathrm{kg} / \mathrm{m}^{2}$ & Median (range) & $22.7(12.4-38.7)$ \\
\hline AST, IU/1 & Median (range) & $47(12-577)$ \\
\hline ALT, IU/1 & Median (range) & $34(9-437)$ \\
\hline$\gamma$-GTP, IU/1 & Median (range) & $41(8-901)$ \\
\hline ALP, IU/1 & Median (range) & $320(117-2193)$ \\
\hline Total bilirubin, $\mathrm{mg} / \mathrm{dl}$ & Median (range) & $1.1(0.2-12.3)$ \\
\hline Albumin, g/dl & Median (range) & $3.4(2.0-4.9)$ \\
\hline Platelet count, $\times 10^{3} / \mu \mathrm{l}$ & Median (range) & $82(21-428)$ \\
\hline Prothrombin time, $\%$ & Median (range) & $75.0(37.6-115.6)$ \\
\hline Glucose, mg/dl & Median (range) & $101(59-296)$ \\
\hline Total cholesterol, mg/dl & Median (range) & $145(73-266)$ \\
\hline Triglyceride & Median (range) & $75(25-373)$ \\
\hline
\end{tabular}

AST: Aspartate aminotransferase; ALT: alanine aminotransferase; $\gamma$ GTP: $\gamma$-glutamyl transpeptidase; ALP: alkaline phosphatase; HBV: hepatitis B virus; HCV: hepatitis C virus; ALD: alcoholic-related liver disease; NASH: nonalcoholic steatohepatitis; $\mathrm{AIH}$; autoimmune hepatitis; PBC: primary biliary cholangitis; IU: international unit.

left leg separately. In the present study, we calculated the total appendicular skeletal muscle mass index (SMI), which was generally used for the evaluation of sarcopenia, and the arm and the leg SMIs which were calculated using the following formulae:

Total SMI $\left(\mathrm{kg} / \mathrm{m}^{2}\right)=$ Total appendicular muscle mass $(\mathrm{kg}) /$ height $(\mathrm{m})^{2}$

Arm SMI $\left(\mathrm{kg} / \mathrm{m}^{2}\right)=$ Total skeletal muscle mass of bilateral upper limbs $(\mathrm{kg}) /$ height $(\mathrm{m})^{2}$

Leg SMI $\left(\mathrm{kg} / \mathrm{m}^{2}\right)=$ Total skeletal muscle mass of bilateral lower limbs $(\mathrm{kg}) /$ height $(\mathrm{m})^{2}$

The Total SMI data were classified into two groups according to the cutoff values of the Japanese guideline for the diagnosis of sarcopenia ( $7.0 \mathrm{~kg} / \mathrm{m}^{2}$ for men and $5.7 \mathrm{~kg} / \mathrm{m}^{2}$ for women) (3). The arm and leg SMI values were classified into two groups according to the median value of each parameter. The state of edema was determined as the ratio of extracellular water (ECW) to total body water (TBW). The degree of overhydration was classified as follows: No overhydration (ECW/TBW $<0.39$ ), mild overhydration $(0.39 \leq \mathrm{ECW} / \mathrm{TBW}<0.40)$, and moderate to severe overhydration $(\mathrm{ECW} / \mathrm{TBW} \geq 0.400)(10)$.
Table II. The data of the three bioimpedance analysis-based indices of skeletal muscle mass index (SMI) for the study patients. Regarding all three indices of SMI, male patients had significantly higher values than female patients $(p<0.001)$. In both genders, the values of total SMI were significantly higher than those of arm SMI $(p<0.001)$ and those of leg SMI $(p<0.001)$. In addition, the values of leg SMI were significantly higher than those of arm SMI $(p<0.001)$.

\begin{tabular}{lcc}
\hline SMI & Subgroup & Median (range), $\mathrm{kg} / \mathrm{m}^{2}$ \\
\hline Total & Male & $7.18(4.41-10.21)$ \\
& Female & $6.03(4.14-7.77)$ \\
Arm & Male & $1.84(0.78-2.83)$ \\
& Female & $1.44(0.84-2.09)$ \\
Leg & Male & $5.33(3.63-8.19)$ \\
& Female & $4.53(3.06-6.83)$ \\
\hline
\end{tabular}

Determination of arm muscle circumference (AMC) and arm muscle area (AMA) based on manual anthropometric measurements. In our Department, the arm circumference (AC) $(\mathrm{cm})$ and triceps skinfold thickness (TSF) (cm) values were measured by a designated dietician as described previously (13). Two commonly used indices of the muscle mass, namely the arm muscle circumference (AMC) and the arm muscle area (AMA), were calculated using the following formulae:

AMC $(\mathrm{cm})=\mathrm{AC}(\mathrm{cm})-\pi \times T S F(\mathrm{~cm})$

AMA $\left(\mathrm{cm}^{2}\right)=[\operatorname{AMC}(\mathrm{cm})]^{2} / 4 \pi$

Based on the standard AC and AMA values for Japanese people (14), the \% AC and \%AMA values were then calculated.

Statistical analyses. The relationships of the arm SMI with the muscle mass indices (\%AMC and \%AMA) obtained by manual anthropometric measurements were determined using Spearman's correlation coefficient. Survival curves were obtained with the Kaplan-Meier method, and the prognoses of the groups were compared using the log-rank test. The differences in the paired ECW/TBW data between the upper and lower limbs were evaluated with Wilcoxon's signed-rank test. For categorical variables, the frequencies in two groups was compared by the chi-squared test. $p$ Values of less than 0.05 were considered to indicate statistical significance.

\section{Results}

Basic clinical characteristics of the patients. The basic clinical characteristics of the enrolled patients are shown in Table I. We studied a total of 353 patients with LC [male $=59.8 \%$; female $=40.2 \%$; median age $=66$ years (range $=24-85$ years)]. The total numbers of patients with hepatitis virus-related cirrhosis and those with non-viral cirrhosis were $257(72.8 \%)$ and 96 (27.2\%), respectively. The data of the BIA-based indices of skeletal muscle mass (total, arm and leg SMIs) are shown in Table II. Regarding all three indices of SMI, male patients had significantly higher values than female patients $(p<0.0001)$. In both 
A

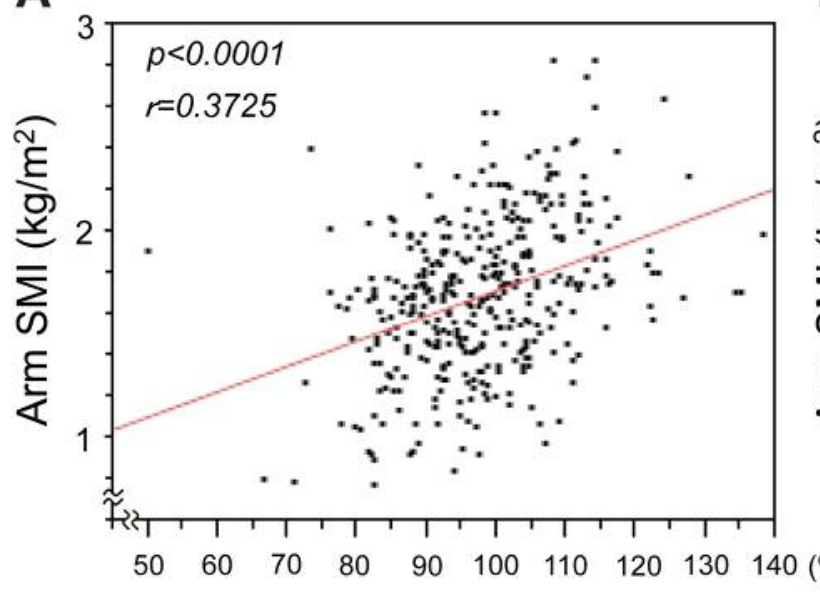

$\% A M C$
B

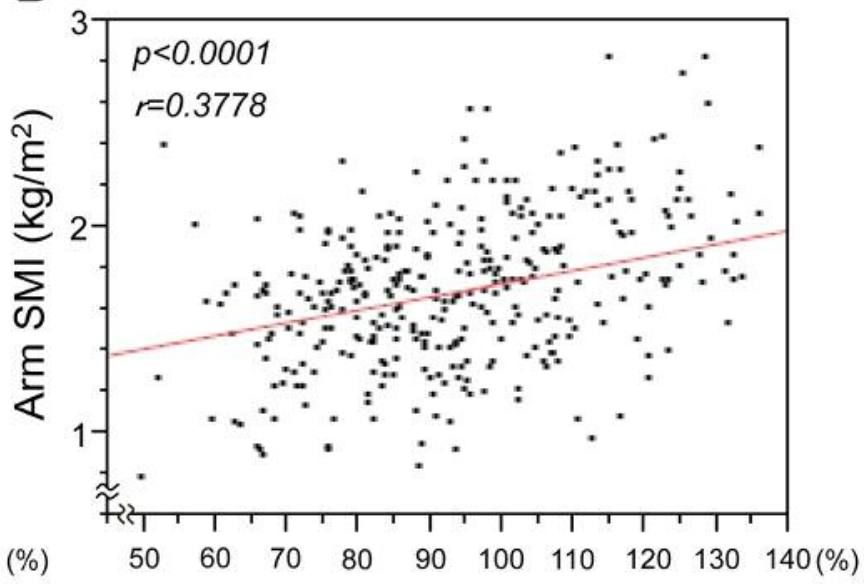

\section{\%AMA}

Figure 1. Correlation of arm skeletal muscle mass index (SMI) with arm muscle circumference (\%AMC) (A) and arm muscle area (\%AMA) (B) values. Anthropometric values were obtained by manual measurements, and the two indices for the muscle mass (\%AMC and \%AMA) were then calculated. The arm SMI values were significantly positively correlated with \%AMC and \%AMA values.

genders, the values of total SMI were significantly higher than those of arm SMI $(p<0.0001)$ and those of leg SMI $(p<0.0001)$. In addition, the values of leg SMI were significantly higher than those of arm SMI $(p<0.0001)$.

Association of the BIA-based arm SMI values with the anthropometric indices of the arm skeletal muscle mass. A previous report suggested that arm SMI was associated with the prognosis of patients with LC (11). Thus, we first evaluated whether the arm SMI was associated with the recognized anthropometric indices of muscle mass (\%AMC and \%AMA), the clinical utility of which had been already reported (15-17). As shown in Figure 1, the arm SMI values positively correlated well with both the \%AMC $(p<0.0001)$ and \%AMA $(p<0.0001)$.

Associations of the SMI values with the prognosis of the patients with cirrhosis. We next evaluated the clinical relevance of the three BIA-based indices of skeletal muscle mass to the prognosis of patients with LC. The mean observation period was 1,557 days (4.27 years). As expected, a low total SMI (indicating sarcopenia), which was calculated using the appendicular skeletal mass, was significantly associated with poorer prognosis $(p=0.0117)$ (Figure 2). When we analyzed the muscle mass data of the upper limbs and that of the lower limbs separately, a low arm SMI was also significantly associated with poorer prognosis of patients with LC ( $p=0.0002)$ (Figure 3A). However, while the patients with a high leg SMI value tended to have a better prognosis, the association was not statistically significant $(p=0.0829)$ (Figure 3B).

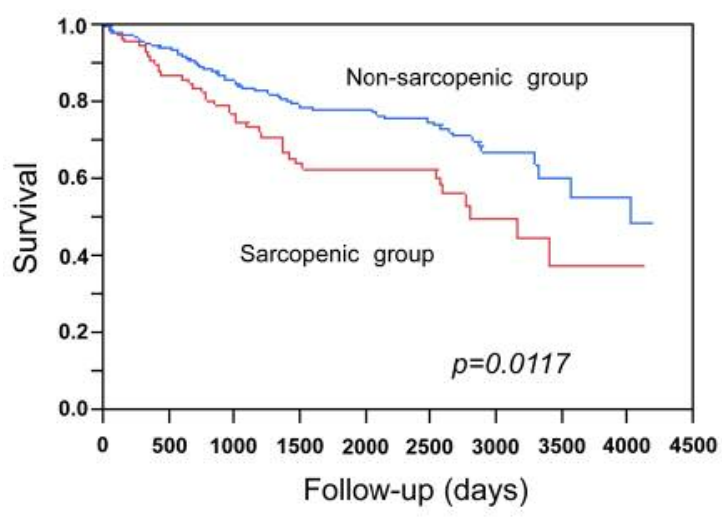

Figure 2. Survival according to total skeletal muscle mass index (SMI). The total SMI was calculated using the bioimpedance analysis-based total appendicular muscle mass. Patients with high total SMI (nonsarcopenic group) had a significantly better survival in comparison to those with low total SMI values (sarcopenic group).

Comparison of overhydration between the upper and lower limbs. Since the presence of edema has been suggested to influence the estimation of the muscle mass, we evaluated the state of overhydration in each limb of the patients with LC. The ECW/TBW values for the lower limbs were significantly higher than those for the upper limbs (Figure 4). When we classified the overhydrated states into three categories, as described in the Patients and Methods section, the frequency of overhydration for the lower limbs was significantly higher than that for the upper limbs (Figure 5). There was no 
A

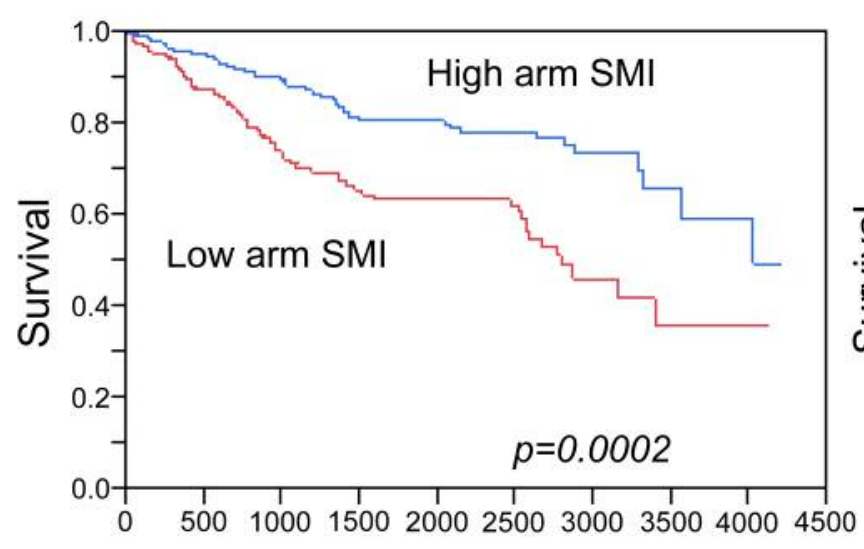

Follow-up (days)
B

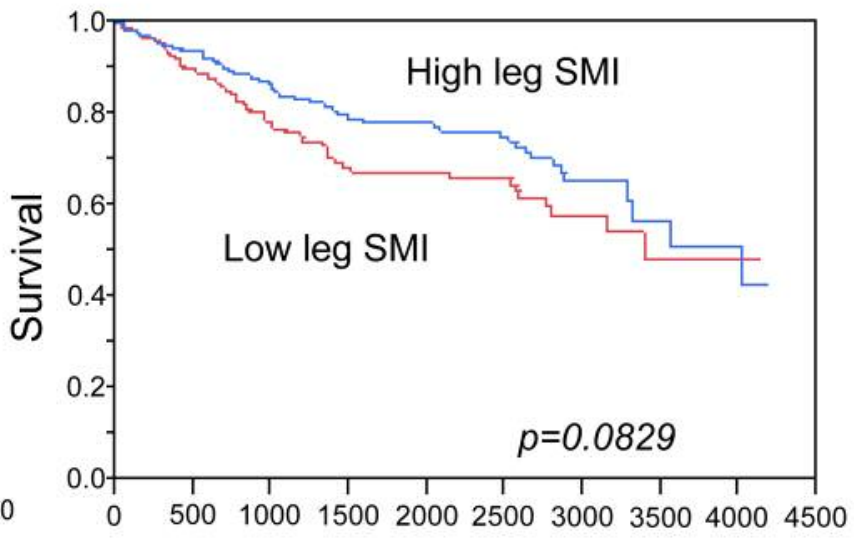

Follow-up (days)

Figure 3. Survival curves according to arm (A) and leg (B) skeletal muscle mass index (SMI). Arm and leg SMI values were calculated using the bioimpedance analysis-based arm skeletal mass and leg muscle mass, respectively. A: Survival was significantly better for patients with high arm SMI values. B: Patients with high leg SMI values tended to have a better prognosis in comparison to those with low values, however, the difference was not statistically significant $(p=0.0829)$.

A

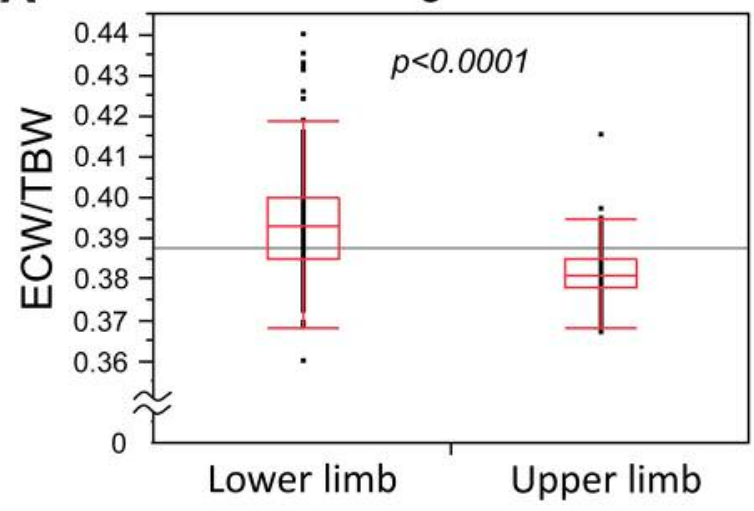

B

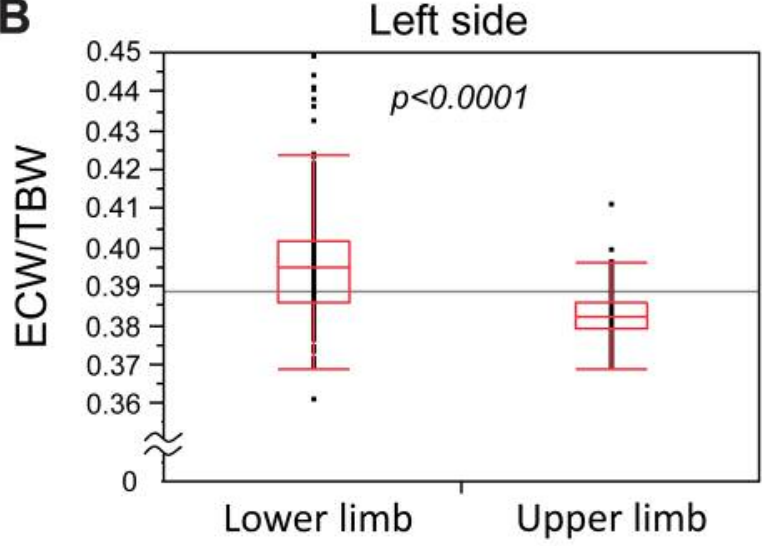

Figure 4. Extracellular water (ECW) to total body water (TBW) values for the lower and upper limbs of the right (A) and left $(B)$ sides of the body. The ECW/TBW values of the lower limbs were significantly higher than those of the upper limbs on both sides of the body. The symbols represent the individual data. The boxes show the interquartile ranges (IQR); the upper lines, middle lines, and the lower lines represent the $75^{\text {th }}$ percentiles, the median values and the $25^{\text {th }}$ percentiles, respectively. The bars show the ranges of the data from the $25^{\text {th }}$ percentile-(1.5XIQR) to the $75^{\text {th }}$ percentile $+(1.5 \times I Q R)$.

significant difference between sides, suggesting that lower limbs are susceptible to oedema and overhydration.

\section{Discussion}

Sarcopenia, which is diagnosed based on the appendicular muscle mass, has been reported to be associated with the prognosis of various diseases. In addition, the results of several physical tests, such as grip strength and walking speed, are included in the diagnostic procedures (3-8). Thus, it is generally considered that both the upper and lower limbs are suitable for evaluating the muscle mass and function. In agreement with these guidelines, we previously reported the value of the SMI determined with the appendicular muscle 
A

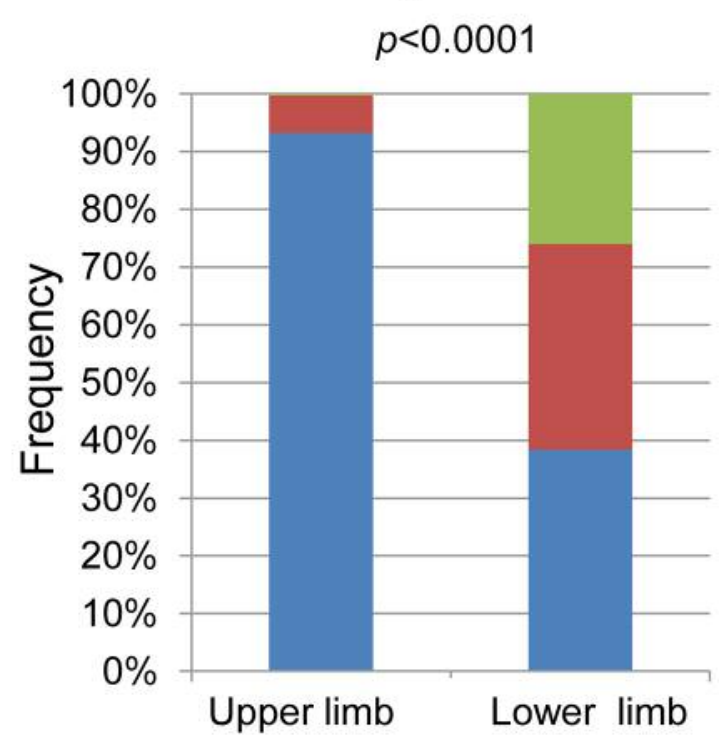

Left side $p<0.0001$

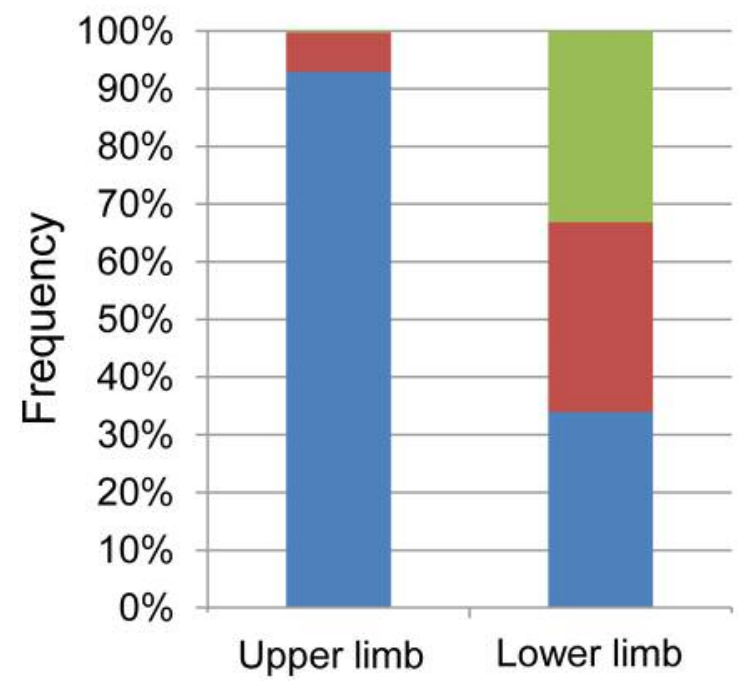

Overhydration

Moderate/severe
Mild
No

Figure 5. Overhydration in the lower and upper limbs. When overhydration was classified into three categories according to the extracellular water $(E C W)$ to total body water $(T B W)$ values (no overhydration: $E C W / T B W<0.39$; mild overhydration: $0.39 \leq E C W / T B W<0.40$; and moderate to severe overhydration: $E C W / T B W \geq 0.400$ ), the frequency of overhydration was significantly higher for the lower limbs than for the upper limbs. There was no significant difference between sides.

mass in relation to the prognosis of patients with cirrhosis (18), and the present study provided a consistent result (Figure 2). However, we were concerned about the effect of water retention/edema on the muscle mass data of patients with cirrhosis, and consequently evaluated the upper and lower limbs separately. Our results suggest that some difference might exist between the upper and lower limbs with regard to the association of the BIA-based SMI values with the prognosis of patients with cirrhosis (Figure 3 ).

It is known that patients with LC are inclined to develop water retention. We previously studied patients with hepatitis virus-related CLDs and reported that overhydration was suggested to increase in severity as the disease progressed (10). According to a report that compared 20 patients with compensated LC and 25 patients with decompensated LC (19), the ECW/TBW ratio of the lower limbs in patients with decompensated cirrhosis was higher than that in those with compensated cirrhosis. However, the ECW/TBW ratios of the upper limbs in these two patient groups did not differ to a statistically significant extent. Consistent with these findings, our results showed that water retention of the lower limbs was higher than that in the upper limbs in the same cases (Figures 4 and 5), suggesting that the muscle mass data for the upper limbs would be less affected by water retention than that for the lower limbs.

It is generally considered that the \% AMC and \% AMA, which are determined by physical assessments, can reflect the muscle mass of the upper limbs with fair to good reproducibility $(15,20)$. In the present study, the BIA-based values of the arm SMI were well correlated with both the $\%$ AMC and \%AMA, which are calculated using directly measured anthropometric data (Figure 1). In addition, the arm SMI value seemed to be better associated with the prognosis in comparison to the leg SMI value in our cohort (Figures 3 and 4). In light of these results, the evaluation of the upper limb muscle mass using the BIA method, which is suggested to be minimally affected by edema, should be clinically beneficial for patients with LC.

The present study was associated with some limitations. Firstly, this was a retrospective study, and patients with various etiologies and follow-up periods were included. Secondly, the study population was limited to 353 patients 
with cirrhosis. Since sarcopenia also occurs in patients without cirrhosis (3), our results should be evaluated in another cohort, particularly a larger cohort that includes patients with noncirrhotic CLD. Thirdly, this study did not evaluate the functional data of the upper and lower limbs (e.g. grip strength and walking speed/chair stand test). In addition, a recent article reported the utility of assessing the lower limb muscles mass in patients with CLD (21). Our cohort was limited to patients with cirrhosis, and the importance of estimating the lower limb muscle mass for the diagnosis of sarcopenia in patients with CLD should be further validated and debated.

In conclusion, despite the tendency for patients with cirrhosis to develop edema/water retention, BIA-based data of the skeletal muscle mass in the upper limbs were suggested to be less affected in comparison to the lower limbs. The estimation of the upper limb muscle mass may be useful for evaluating the skeletal muscle mass in patients with cirrhosis.

\section{Funding}

This work was partly granted by Hyogo Innovative Challenge, Hyogo College of Medicine, Japan.

\section{Conflicts of Interest}

The Authors declare no conflicts of interest in association with the present study.

\section{Authors' Contributions}

YS collected and analyzed the data; HE designed the study, analyzed the data and wrote the article. KK collected and analyzed the data. EM, NH, TN, YI and HI collected the data; SN collected the data and supervised the study. All the Authors reviewed and edited the article and approved the final version.

\section{Acknowledgements}

The Authors are grateful to S Inui, Y Higuchi, N Kanazawa, Y Matsushita, S Fujii, H Kido and K Minemoto (Hyogo College of Medicine) for their technical and secretarial assistance.

\section{References}

1 Rosenberg IH: Sarcopenia: origins and clinical relevance. J Nutr 127(5 Suppl): 990S-991S, 1997. PMID: 9164280. DOI: 10.1093/jn/127.5.990S

2 Cruz-Jentoft AJ and Sayer AA: Sarcopenia. Lancet 393(10191): 2636-2646, 2019. PMID: 31171417. DOI: 10.1016/S01406736(19)31138-9

3 Nishikawa H, Shiraki M, Hiramatsu A, Moriya K, Hino K and Nishiguchi S: Japan Society of Hepatology guidelines for sarcopenia in liver disease (1st edition): Recommendation from the Working Group for Creation of Sarcopenia Assessment Criteria. Hepatol Res 46(10): 951-963, 2016. PMID: 27481650. DOI: $10.1111 /$ hepr.12774
4 Fielding RA, Vellas B, Evans WJ, Bhasin S, Morley JE, Newman AB, Abellan van Kan G, Andrieu S, Bauer J, Breuille D, Cederholm T, Chandler J, De Meynard C, Donini L, Harris T, Kannt A, Keime Guibert F, Onder G, Papanicolaou D, Rolland Y, Rooks D, Sieber C, Souhami E, Verlaan S and Zamboni M: Sarcopenia: An undiagnosed condition in older adults. Current consensus definition: Prevalence, etiology, and consequences. International working group on sarcopenia. J Am Med Dir Assoc 12(4): 249-256, 2011. PMID: 21527165. DOI: 10.1016/j.jamda.2011.01.003

5 Studenski SA, Peters KW, Alley DE, Cawthon PM, McLean RR, Harris TB, Ferrucci L, Guralnik JM, Fragala MS, Kenny AM, Kiel DP, Kritchevsky SB, Shardell MD, Dam TT and Vassileva MT: The FNIH sarcopenia project: Rationale, study description, conference recommendations, and final estimates. J Gerontol A Biol Sci Med Sci 69(5): 547-558, 2014. PMID: 24737557. DOI: 10.1093/gerona/glu010

6 Chen LK, Liu LK, Woo J, Assantachai P, Auyeung TW, Bahyah KS, Chou MY, Chen LY, Hsu PS, Krairit O, Lee JS, Lee WJ, Lee Y, Liang CK, Limpawattana P, Lin CS, Peng LN, Satake S, Suzuki T, Won CW, Wu CH, Wu SN, Zhang T, Zeng P, Akishita $\mathrm{M}$ and Arai H: Sarcopenia in Asia: Consensus report of the Asian Working Group for Sarcopenia. J Am Med Dir Assoc 15(2): 95-101, 2014. PMID: 24461239. DOI: 10.1016/j.jamda. 2013.11.025

7 Cruz-Jentoft AJ, Baeyens JP, Bauer JM, Boirie Y, Cederholm T, Landi F, Martin FC, Michel JP, Rolland Y, Schneider SM, Topinková E, Vandewoude M, Zamboni M; European Working Group on Sarcopenia in Older People: Sarcopenia: European consensus on definition and diagnosis: Report of the European Working Group on Sarcopenia in Older People. Age Ageing 39(4): 412-423, 2010. PMID: 20392703. DOI: 10.1093/ageing/ afq034

8 Cruz-Jentoft AJ, Bahat G, Bauer J, Boirie Y, Bruyère O, Cederholm T, Cooper C, Landi F, Rolland Y, Sayer AA, Schneider SM, Sieber CC, Topinkova E, Vandewoude M, Visser M, Zamboni M; Writing Group for the European Working Group on Sarcopenia in Older People 2 (EWGSOP2), and the Extended Group for EWGSOP2: Sarcopenia: Revised European consensus on definition and diagnosis. Age Ageing 48(1): 16-31, 2019. PMID: 30312372. DOI: 10.1093/ageing/afy 169.

9 Ianni Filho D, Boin IFSF and Yamanaka A: Bioimpedance: New approach to non-invasive detection of liver fibrosis - a pilot study. Arq Gastroenterol 55(1): 2-6, 2018. PMID: 29561971. DOI: 10.1590/S0004-2803.201800000-02

10 Nishikawa H, Yoh K, Enomoto H, Ishii N, Iwata Y, Nakano C, Takata R, Nishimura T, Aizawa N, Sakai Y, Ikeda N, Hasegawa K, Takashima T, Iijima $\mathrm{H}$ and Nishiguchi S: Extracellular water to total body water ratio in viral liver diseases: A study using bioimpedance analysis. Nutrients 10(8): pii: E1072, 2018. PMID: 30103528. DOI: 10.3390/nu 10081072

11 Iwasa M, Hara N, Terasaka E, Hattori A, Ishidome M, MifujiMoroka R, Miyachi H, Sugimoto R, Tanaka H, Fujita N, Kobayashi Y, Iwata $\mathrm{K}$ and Takei $\mathrm{Y}$ : Evaluation and prognosis of sarcopenia using impedance analysis in patients with liver cirrhosis. Hepatol Res 44(10): E316-317, 2014. PMID: 25257689. DOI: $10.1111 /$ hepr.12217

12 Enomoto H, Ueno Y, Hiasa Y, Nishikawa H, Hige S, Takikawa Y, Taniai M, Ishikawa T, Yasui K, Takaki A, Takaguchi K, Ido A, Kurosaki M, Kanto T, Nishiguchi S; Japan Etiology of Liver 
Cirrhosis Study Group in the $54^{\text {th }}$ Annual Meeting of JSH: Transition in the etiology of liver cirrhosis in Japan: a nationwide survey. J Gastroenterol, 2019. PMID: 31768801. DOI: 10.1007/s00535-019-01645-y

13 Moriwaki E, Enomoto H, Saito M, Hara N, Nishikawa H, Nishimura $\mathrm{T}$, Iwata $\mathrm{Y}$, Iijima $\mathrm{H}$ and Nishiguchi $\mathrm{S}$ : Anthropometric assessment with the bioimpedance method is associated with the prognosis of cirrhotic patients. In Vivo 34(2): XXX-XXX, 2020.

14 Hosoya N: Japanese anthropometric reference data 2001 (JARD 2001). Jpn J Nutr Assess 19(Suppl.): 1-81, 2002.

15 Madden AM and Smith S: Body composition and morphological assessment of nutritional status in adults: a review of anthropometric variables. J Hum Nutr Diet 29(1): 7-25, 2016. PMID: 25420774. DOI: $10.1111 / \mathrm{jhn} .12278$

16 Santos LAA, Lima TB, Ietsugu MDV, Nunes HRC, Qi X and Romeiro FG: Anthropometric measures associated with sarcopenia in outpatients with liver cirrhosis. Nutr Diet 76(5): 613-619. 2019. PMID: 30869199. DOI: 10.1111/1747-0080.12523

17 Lucidi C, Lattanzi B, Di Gregorio V, Incicco S, D’Ambrosio D, Venditti M, Riggio O and Merli M: A low muscle mass increases mortality in compensated cirrhotic patients with sepsis. Liver Int 38(5): 851-857, 2018. PMID: 29323441. DOI: 10.1111/liv.13691

18 Nishikawa H, Enomoto H, Iwata Y, Nishimura T, Iijima H and Nishiguchi S: Clinical utility of bioimpedance analysis in liver cirrhosis. J Hepatobiliary Pancreat Sci 24(7): 409-416, 2017. PMID: 28371518. DOI: 10.1002/jhbp.455
19 Davenport A, Argawal B, Wright G, Mantzoukis K, Dimitrova $\mathrm{R}$, Davar J, Vasianopoulou $\mathrm{P}$ and Burroughs AK: Can noninvasive measurements aid clinical assessment of volume in patients with cirrhosis? World J Hepatol 5(8): 433-438, 2013. PMID: 24023982. DOI: 10.4254/wjh.v5.i8.433

20 Hasse J, Strong S, Gorman MA and Liepa G: Subjective global assessment: alternative nutrition-assessment technique for livertransplant candidates. Nutrition 9(4): 339-343, 1993. PMID: 8400590.

21 Hiraoka A, Izumoto H, Ueki H, Yoshino T, Aibiki T, Okudaira T, Yamago H, Suga Y, Iwasaki R, Tomida H, Mori K, Miyata H, Tsubouchi E, Kishida M, Ninomiya T, Hirooka M, Abe M, Matsuura B, Hiasa Y and Michitaka K: Easy surveillance of muscle volume decline in chronic liver disease patients using finger-circle (Yubi-Wakka) test. J Cachexia Sarcopenia Muscle 10(2): 347-354, 2019. PMID: 30793533. DOI: 10.1002/jcsm. 12392
Received January 10, 2020

Revised January 24, 2020

Accepted January 29, 2020 